\title{
COMPUTATIONAL AND EXPERIMENTAL STUDY ON RING TONE
}

\author{
KAZUO MATSUURA ${ }^{1}$, KOH MUKAI $^{1} \&$ MIKAEL ANDERSEN LANGTHJEM ${ }^{2}$ \\ ${ }^{1}$ Graduate School of Science and Engineering, Ehime University, Japan \\ ${ }^{2}$ Department of Engineering, Aarhus University, Denmark
}

\begin{abstract}
Sound produced when jets issued from a circular nozzle collide with a downstream ring coaxial with the jet is called ring tone. The ring tone is investigated through experiments and direct sound computations. The inner diameters of the circular nozzle exit and the ring are both $30 \mathrm{~mm}$. In the experiments, the frequency spectra of the ring tone are measured for various impingement lengths of $20-40 \mathrm{~mm}$ and jet velocities of 5-15 m/s. The tone is also compared with the hole tone, in which the downstream ring is replaced with a plate with a hole. As a general trend, the peak sound intensity shifts to higher frequency as the jet speed is increased in both tones. Multiple series of peaks are observed for each impingement length. While the peaks generally become higher with increasing jet speed, the amplification is not necessarily monotonic. When the ring tone is compared with the hole tone, the peaks are much lower and the frequency distribution is broader. Regarding the variation of the dominant ring-tone and hole-tone peak frequencies with jet speed, differences between the ring tone and hole tone appear at the locations of mode jumps. In the computations, the onset of self-sustained feedback oscillations in the ring tone is clarified from the view point of the throttling mechanism originally proposed for the hole tone, i.e., the coupling between the mass flow through the ring, vortex impingement and global pressure fluctuation.
\end{abstract}

Keywords: ring tone, hole tone, aeroacoustics, direct sound computation, wind tunnel.

\section{INTRODUCTION}

Ring tone is a kind of feedback sound emitted from a system in which pressure waves generated when vortices from a nozzle collide with a downstream obstacle, propagate upstream, and regulate the timing of further vortex ejection from the nozzle [1]-[4]. We encounter feedback tones in many practical situations such as solid propellant rocket motors, automobile intake and exhaust systems, ventilation systems, gas distribution systems, and whistling kettles.

Ring tone is a dipole sound with an efficiency scaling of the third power of the jet Mach number; the dipole strength is equal to the unsteady force on the ring [4]. These dipoles radiate in the direction of the jet axis [3]. Chanaud and Powell [5] investigated ring tone with a torus with an inner diameter of $5 \mathrm{~mm}$ formed from copper wire with a diameter of $1.5 \mathrm{~mm}$. Data were taken by varying the edge distance at constant Reynolds number and by varying the Reynolds number at constant edge distance. They found that at large spacing ratios, the hole- and ring-tone minimum-edge-distance contours have similar shapes and are distinct from an edge-tone contour. At small spacing ratios, the minimum-edge-distance contour for the ring-tone system deviates markedly from other contours, there being folds in the contours. The folds are seen to be related to jumps at higher Reynolds numbers. Obata et al. [6] experimentally investigated the ring tone. They found that multiple frequency components have substantial amplitudes compared to the fundamental frequency component, and showed that the lowest frequency component can interact with the fundamental component either to reinforce itself or to produce an additional frequency component.

Although edge and hole tones have been investigated relatively in detail [3], [7]-[10] among feedback sounds, the details of ring tones, such as flow fields and the difference 
between the ring and hole tones, are unknown except for the description mentioned above. Therefore, in this study, a ring tone system is developed, the frequency spectra of the ring tone are measured for various impingement lengths and jet velocities, and the sound generation mechanism is investigated through a direct sound computation. In the measurements, the ring tone is compared with the hole tone, which is generated when a metal plate with a hole with the same diameter as the nozzle outlet is installed instead of the ring with the ring tone. To our best knowledge, this study is the first case of applying a direct computation to the ring tone.

In Section 2, the experimental methods are described including the geometries of the ring and hole, the wind tunnel, flow conditions, and the measurement system. Section 3 describes the numerical methods, computational conditions, grids, and initial and boundary conditions. In Section 4, experimental results are shown such as frequency spectra and the variation of the dominant peak frequency for various jet speeds and impingement lengths. The experimental results for the ring tone are compared with those for the hole tone. Section 5 discusses the relationship between vortex impingement on the ring, mass flow variation through the ring, and global pressure variation in the flow field between the nozzle exit and the ring.

\section{EXPERIMENTAL METHODS}

Experiments were conducted to gain an insight into the sound properties of the ring tone. Fig. 1 shows the experimental system with a ring placed downstream of the nozzle. A jet issued from the circular nozzle of a wind tunnel, and the ring was coaxial to the nozzle. The inner diameters of the nozzle exit $d_{0}$ and the ring were both $30 \mathrm{~mm}$. The outer diameter of the ring was $40 \mathrm{~mm}$. The distance between the nozzle exit and the ring, the impingement length $L_{\mathrm{im}}$ was varied from 20 to $40 \mathrm{~mm}$. Sound pressure was measured at $r=120 \mathrm{~mm}$ for $\mathrm{z}=L_{\mathrm{im}} / 2$ with a condenser microphone. Data were passed to an FFT analyser. The ring was acoustically compact. The speed of the circular jet was varied from 5 to $15 \mathrm{~m} / \mathrm{s}$. At $20^{\circ} \mathrm{C}$, these velocities correspond to Reynolds numbers $\left(R e=u_{0} d_{0} / v\right)$ of $9.96 \times 10^{3}-2.98 \times 10^{4}$. Fig. 2 shows the overview and schematic of the wind tunnel. The circular jets were generated by a smooth circular contraction of airflow from the almost axisymmetric wind tunnel.

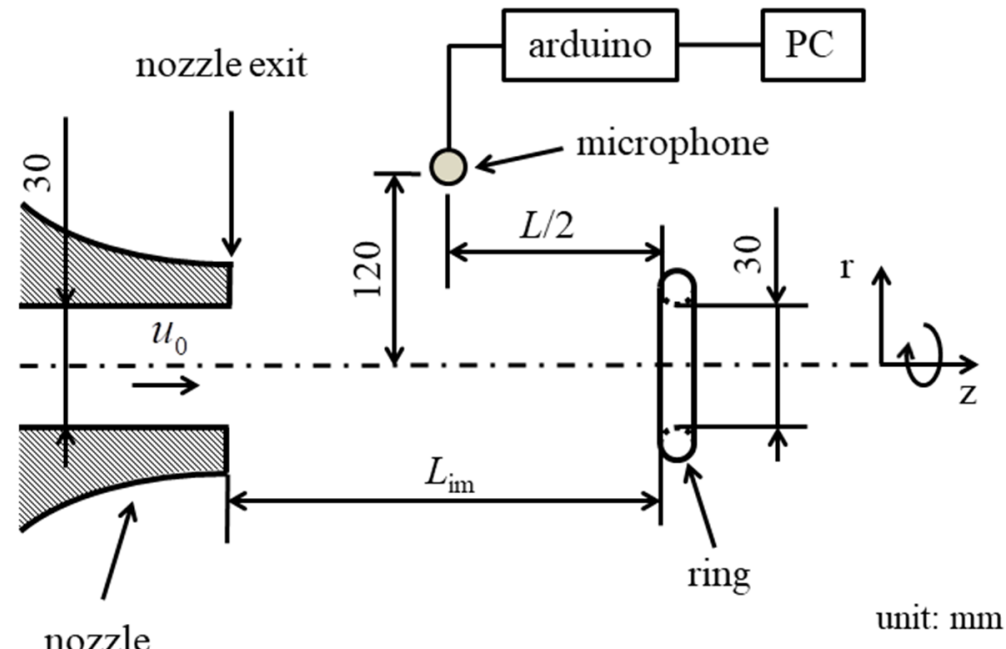

Figure 1: Experimental system. 


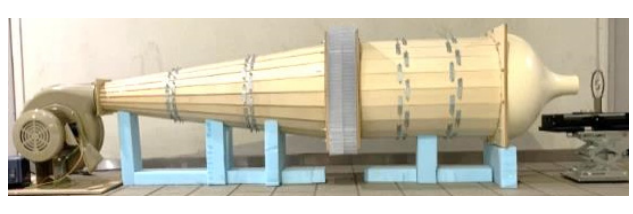

(a)

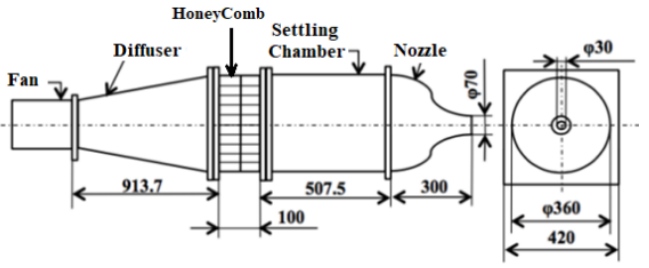

(b)

Figure 2: Wind tunnel. (a) Photo; and (b) Schematic; unit: mm.

In addition to the experiments on the ring tone, experiments on the hole tone were conducted to compare the sound properties of the two tones because the hole tone is similar and more details are known about it. Fig. 3 shows the fabricated ring and hole plate and their schematics. To fix the ring in the air, the ring was pulled by six piano wires each with a diameter of $0.25 \mathrm{~mm}$.

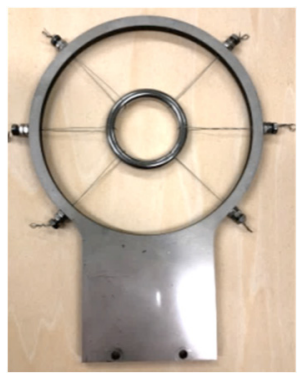

(a)

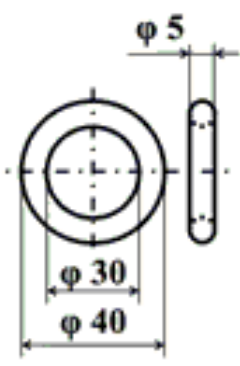

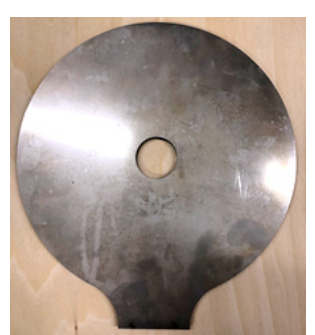

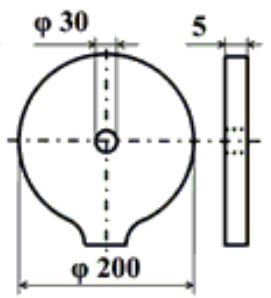

(b)

Figure 3: Ring and hole plate; unit: $\mathrm{mm}$. The inner and outer diameters of the ring are $30 \mathrm{~mm}$ and $40 \mathrm{~mm}$, respectively. The diameter of the hole is $30 \mathrm{~mm}$, and the thickness is $5 \mathrm{~mm}$. (a) Fabricated ring (left: photo; right: schematic); and (b) Fabricated hole plate (left: photo; right: schematic).

\section{NUMERICAL METHODS}

A direct sound computation was conducted to clarify the onset of the self-sustained oscillation of the ring tone. Only near-field behaviours were considered because the interaction of a flow with the ring and the associated feedback propagation of pressure to the nozzle exit is the central concern of the computation.

The governing equations are the unsteady three-dimensional compressible Navier-Stokes equations. The ideal gas law closes the system of the equations. The equations were solved with the finite-difference method. Spatial derivatives that appear in metrics, convective and viscous terms were evaluated with the 6th-order tridiagonal compact scheme [11]. Near the boundaries, the 4th-order one-sided classical Padé scheme was used at one point inside the boundaries. Time-accurate solutions to the governing equations were obtained using the 3rdorder Runge-Kutta scheme. In addition to the spatial discretisation and time integration, 10th-order implicit filtering [12] was introduced to suppress numerical instabilities that arise 
from the central differencing in the compact scheme. The parameter on the left-hand side, which is associated with filtering strength, was set to be 0.492. An implicit 4th-order filter was used near the boundaries. This method has been well validated for predicting the original hole tone [9], [10].

Fig. 4 shows the structured mesh used for the computation. Every five grid lines are drawn for clarity. There were a total of approximately $5.2 \mathrm{M}$ grid points. The overall mesh consisted of four zones: the inlet nozzle, the exterior domain of the nozzle, the ring zone, and the background space. Each zone is shown in a different colour. The ring in the computational domains was represented with the overset method. The outer boundaries of the ring zone were interpolated from the background space at each time step. The boundaries of the blank region of the background space were interpolated from the ring zone. Trilinear interpolation was used in this study. The $z$-axis was defined in the streamwise (axial) direction. Near the walls, the first mesh widths in the normal directions were $8.33 \times 10^{-4} d_{0}$. The streamwise widths of the background mesh around the ring were approximately $3.28 \times 10^{-2} d_{0}$. The far boundaries in the $z$ and $r$ directions were located at $70.36 d_{0}$ and $70.86 d_{0}$, respectively. The inlet boundary of the inlet nozzle was located at $\mathrm{z}=-0.6 d_{0}$.

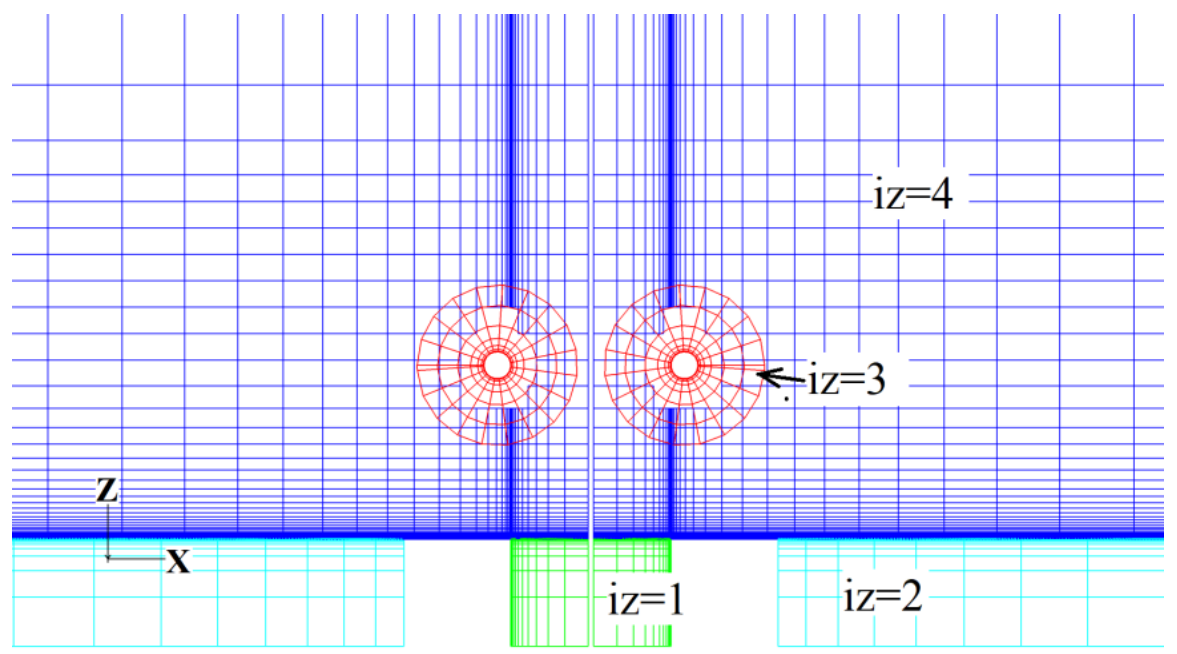

Figure 4: Computational mesh for the ring tone (axisymmetric). Zones iz $=1, \ldots, 4$ are the nozzle inlet, the exterior domain of the nozzle, the ring zone, and the background space, respectively. Every five grid lines are drawn for clarity.

\section{EXPERIMENTAL RESULTS}

The frequency spectra of the ring tone were measured for various impingement lengths and jet velocities [13]. Similar measurements were conducted also for the hole tone to clarify the differences between the ring and hole tones.

Fig. 5 shows the results. As a general trend, the peak sound intensity shifts to higher frequency with increasing jet speed in both tones. Multiple series of peaks are observed for $L_{\mathrm{im}}$ in both tones. While the peak sound intensity generally becomes stronger with increasing jet speed, the amplification is not monotonic. When the ring tone is compared with the hole tone, the peak sound intensities are much lower and the frequency distribution is broader. The lower intensity of the ring tone is consistent with [14]. 


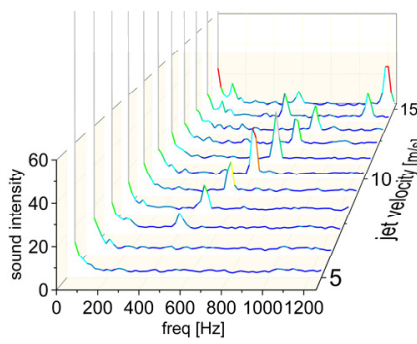

(i) $L_{\mathrm{im}}=20 \mathrm{~mm}$

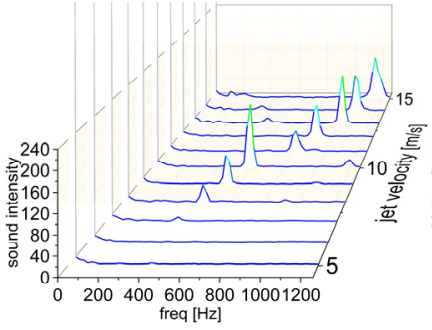

(i) $L_{\mathrm{im}}=20 \mathrm{~mm}$

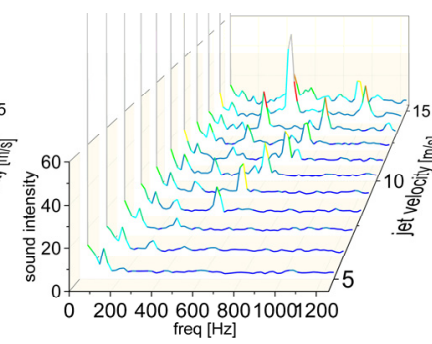

(ii) $L_{\text {im }}=30 \mathrm{~mm}$

(a)

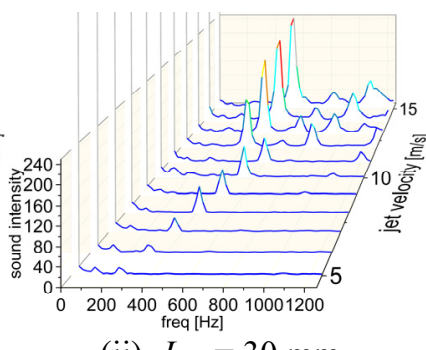

(ii) $L_{\text {im }}=30 \mathrm{~mm}$

(b)

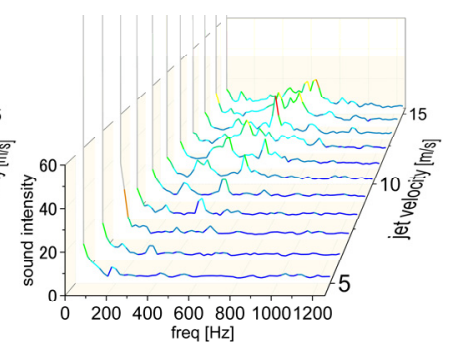

(iii) $L_{\text {im }}=40 \mathrm{~mm}$

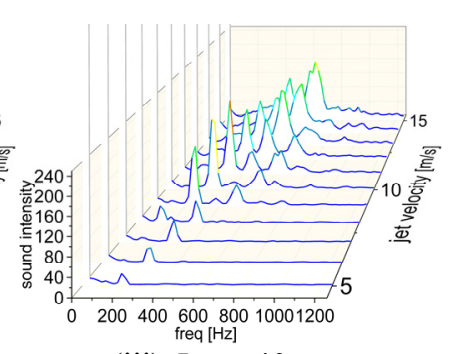

(iii) $L_{\text {im }}=40 \mathrm{~mm}$

Figure 5: Frequency spectra for various impingement lengths and jet speeds. (a) Ring tone; and (b) Hole tone.

Fig. 6 shows the variation of the dominant ring-tone and hole-tone peak frequencies with jet speed for $L_{\mathrm{im}}=20,30$ and $40 \mathrm{~mm}$. In the figure, correlations based on Rossiter's equations $n / f=L_{\mathrm{im}} / u_{\mathrm{c}}+L_{\mathrm{im}} / c_{0}[15]$ are also plotted. Here, $n, f, L_{\mathrm{im}}$, and $c_{0}$ are the stage index, frequency, impingement length, and speed of sound, respectively; $u_{\mathrm{c}}=0.6 u_{0}$ is the convection velocity. As with the computational results in Fig. 5, the peak frequencies generally increase linearly. Differences between the ring tone and the hole tone appear at the locations of mode jumps, i.e., jumps in the stage index. Both integer stage indices $(n)$ and fractional indices appear.

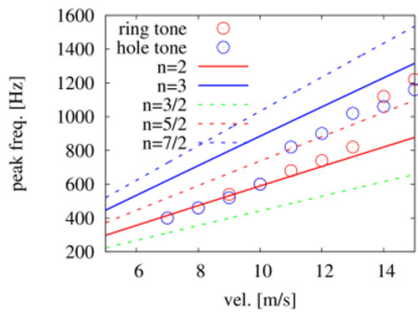

(a)

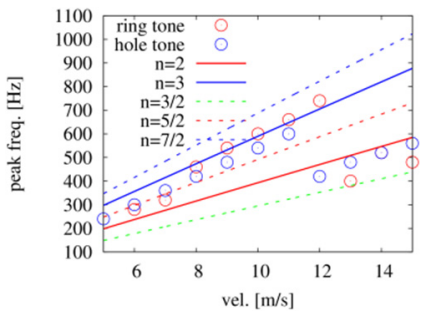

(b)

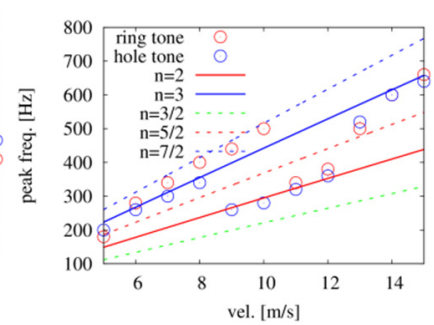

(c)

Figure 6: Variation of the dominant peak frequency with jet speed for various impingement lengths for the ring tone and hole tone. (a) $L_{\mathrm{im}}=20 \mathrm{~mm}$; (b) $L_{\mathrm{im}}=$ $30 \mathrm{~mm}$; and (c) $L_{\mathrm{im}}=40 \mathrm{~mm}$. 
Fig. 7 shows vortex structures and the divergence of velocity at different times. The vortical structures are visualised with the iso-surfaces of the second invariance of the velocity gradient tensor for $Q=1 \times 10^{-5} . t$ denotes the elapsed time from the initial condition, and $t_{\mathrm{d}}$ is the time corresponding to 2,500 computational time steps. $t=0$ corresponds to the initial condition with quiescent air in the flow field except for the nozzle inlet, where a Pohlhausentype laminar velocity profile is imposed. The boundary layer thickness is assumed to be $1 \mathrm{~mm}$. Velocities and lengths are non-dimensionalised with reference quantities $v_{\text {ref }}$ and $l_{\text {ref }}$, respectively. In this study, $v_{\text {ref }}=340 \mathrm{~m} / \mathrm{s}$ and $l_{\text {ref }}=1 \mathrm{~mm}$. Vortices shed from the nozzle exit collide with the ring. When the vortices pass through the ring, new vortices are generated.

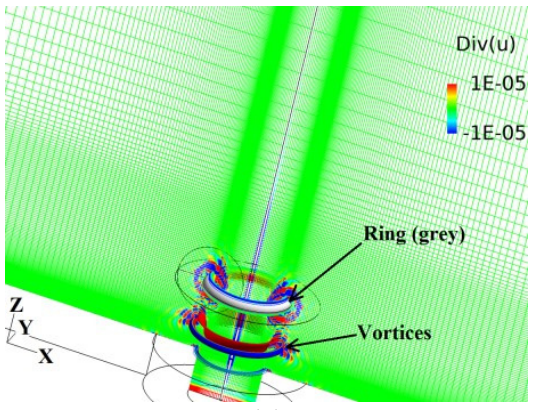

(a)

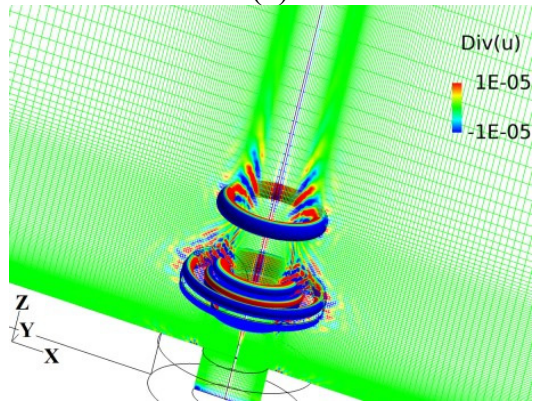

(c)

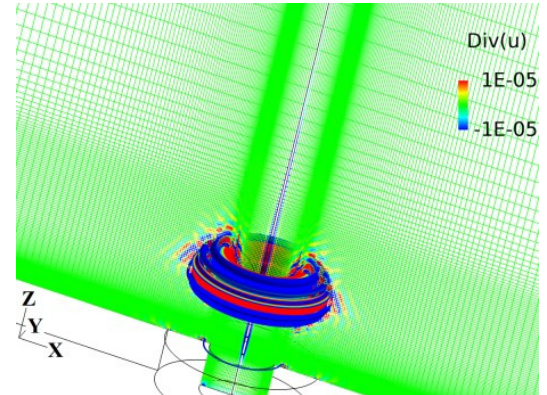

(b)

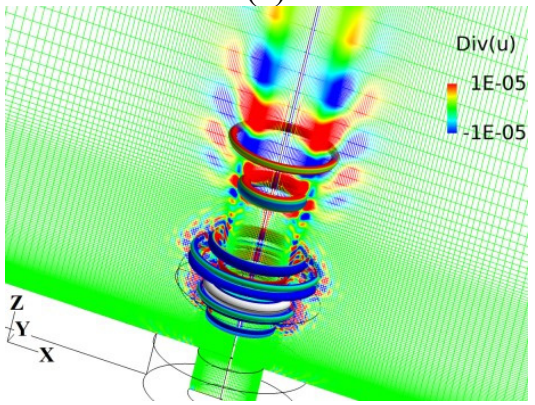

(d)

Figure 7: Vortical structures and divergence of velocity. The vortical structures are visualised with iso-surfaces of the second invariance of the velocity gradient tensor for $Q=1 \times 10^{-5}$. (a) $t / t_{\mathrm{d}}=34$; (b) $t / t_{\mathrm{d}}=80$; (c) $t / t_{\mathrm{d}}=126$; and (d) $t / t_{\mathrm{d}}=$ 174.

To show the interaction of vortices with the ring more clearly, Fig. 8 shows the distribution of $\omega_{y}$ in the $y=0$ plane. Here, the $y$ direction is normal to the sheet. At $t / t_{\mathrm{d}}=34$, a vortex ring is convected along the circular jet and located between the nozzle exit and the ring. At $t / t_{\mathrm{d}}=80$, the initial vortex ring collides with the ring, and secondary vortices, i.e., the wakes of the ring, are generated. After the vortices pass through the ring, some vortices are convected rapidly, and other vortices stay around the ring for a while and are convected afterward.

The transient process from the initial state to the oscillatory states was analysed to investigate the onset of the feedback oscillation. As clarified in [9], the hole tone is governed 


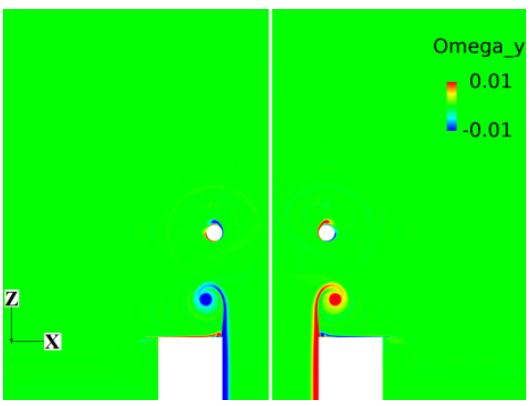

(a)

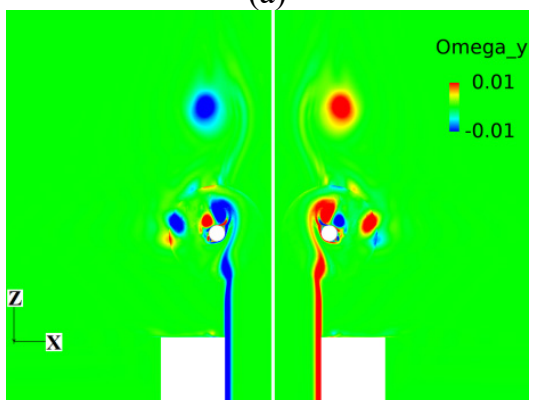

(c)

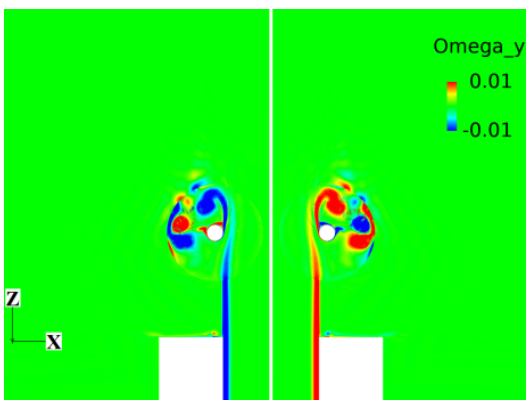

(b)

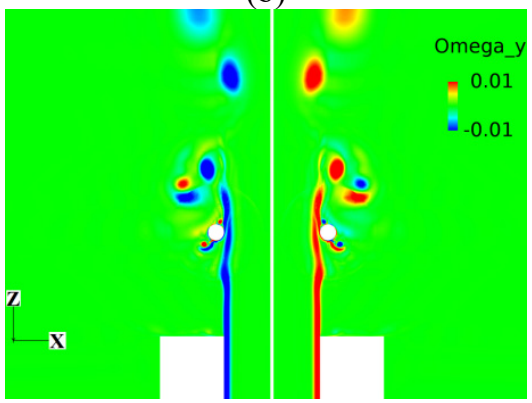

(d)

Figure 8: Distribution of $\omega_{y}$ on $y=0$ plane. (a) $t / t_{\mathrm{d}}=34$; (b) $t / t_{\mathrm{d}}=80$; (c) $t / t_{\mathrm{d}}=126$; and (d) $t / t_{\mathrm{d}}=174$.

by the axisymmetric throttling mechanism, which links mass flow through the hole, vortex impingement and global pressure fluctuation. The ring tone was analysed from the same viewpoint.

Fig. 9 shows the time variation of the mass flow through the ring and gauge pressure sampled at $\mathrm{P}_{1}[(r, z)=(15,0.126)], \mathrm{P}_{2}[(7.46,0.126)]$, and $\mathrm{P}_{3}[(22.5,0.126)]$. The axial position of the mass flow evaluation is the centre of the ring. Initially, the mass flow increases monotonically until around $t / t_{\mathrm{d}}=72$. Oscillations in the mass flow appear afterwards. Peaks are observed at $t / t_{\mathrm{d}}=72,119$, and 145, and valleys are observed at $t / t_{\mathrm{d}}=95,134$, and 155 . Except for the initial peak around $t / t_{\mathrm{d}}=56$, the peaks and valleys of the gauge pressure approximately coincide with those of the mass flow variation, which means that mass flow variation induces the pressure oscillations. The time for sound propagation over $L_{\mathrm{im}}=30 \mathrm{~mm}$ is $\Delta t / t_{\mathrm{d}}=0.625$. Therefore, the time lag of the pressure fluctuation between the ring and the nozzle exit is negligible.

To clarify velocity fields causing the mass flow variation through the ring, Fig. 10 shows the distributions at the peaks and valleys of circumferentially-averaged $z$-directional and $r$ directional velocities along the radial position from the rotational axis. The velocity vector $v$ at a point is decomposed into the radial $(r)$, axial $(z)$, and circumferential $(\theta)$ components using cylindrical coordinates: $v=v_{r} \boldsymbol{e}_{r}+v_{\theta} \boldsymbol{e}_{\theta}+v_{z} \boldsymbol{e}_{z}$, where $\boldsymbol{e}_{r}, \boldsymbol{e}_{\theta}$, and $\boldsymbol{e}_{z}$ are the unit normal basis vectors. The downstream direction of the rotational axis is taken as the positive $z$ direction, and $\boldsymbol{e}_{\mathrm{z}}$ and $\boldsymbol{e}_{\mathrm{r}}$ are taken in the positive $z$ direction and outward directions, respectively. The circumferential direction is then defined according to a right-handed system. 


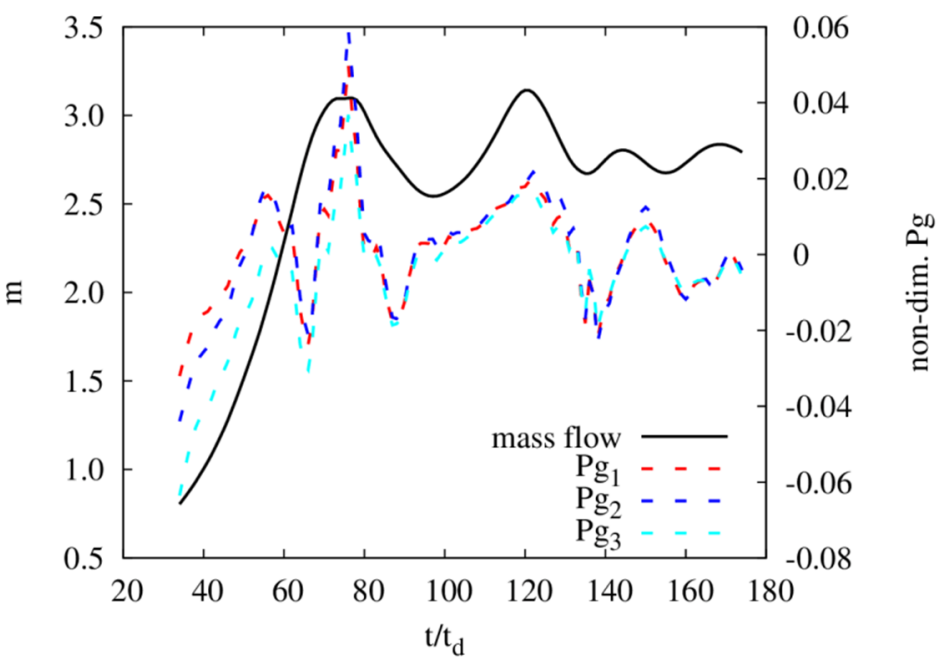

Figure 9: Time variation of mass flow through the ring $m$ and gauge pressure $\mathrm{P}_{\mathrm{g}}$. The subscripts of the gauge pressure denote sampling locations. $\mathrm{P}_{1}:(r, z)=$ $(15,0.126) ; \mathrm{P}_{2}:(7.46,0.126) ; \mathrm{P}_{3}:(22.5,0.126)$.

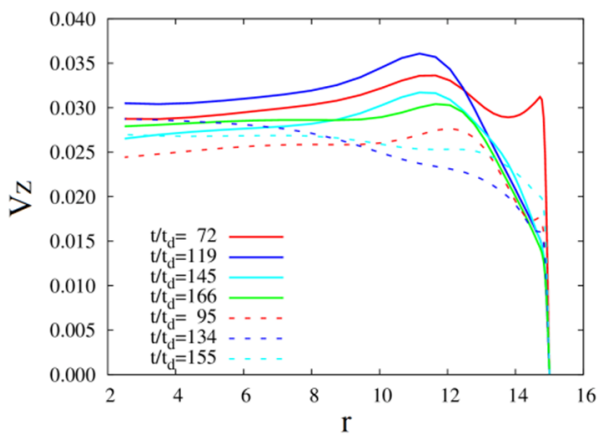

(a)

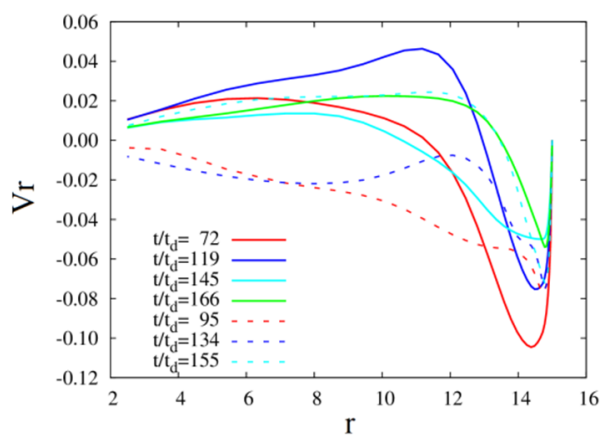

(b)

Figure 10: Circumferentially-averaged velocities in the axial $(z)$ and radial $(r)$ directions. $r$ is the radial distance from the rotational axis. (a) $z$-directional velocity; and (b) $r$-directional velocity.

At the peaks, $v_{\mathrm{z}}$ and $v_{\mathrm{r}}$ are generally higher than at the valleys. The rapid drops in $v_{\mathrm{z}}$ and $v_{\mathrm{r}}$ near $r=d_{0} / 2$ mean the existence of a wall. Positive $v_{\mathrm{r}}$ means that flow vectors point outward when the flow passes through the ring, and negative $v_{\mathrm{r}}$ means that flow vectors point inward. Thus, the periodic variation of flow vectors causes mass flow variation through the ring. Fig. 11 shows the distribution of $\omega_{\mathrm{y}}$ when the mass flows are the peaks and valleys. As observed in Fig. 11(a), the bulged regions denoted by the black arrows leave the ring when the mass flows become peaks. When the mass flows become valleys, the bulged regions collide with the ring as found in Fig. 11(b). 


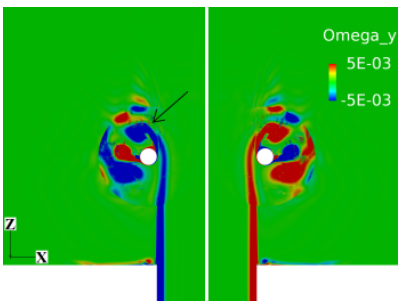

(i) $t / t_{\mathrm{d}}=72$

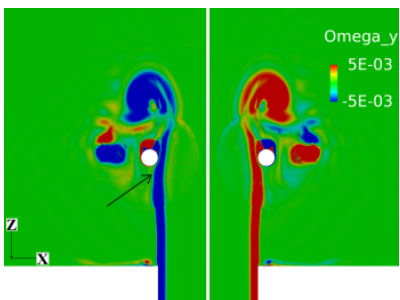

(i) $t / t_{\mathrm{d}}=95$

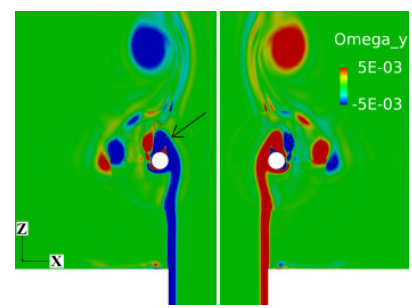

(ii) $t / t_{\mathrm{d}}=119$

(a)

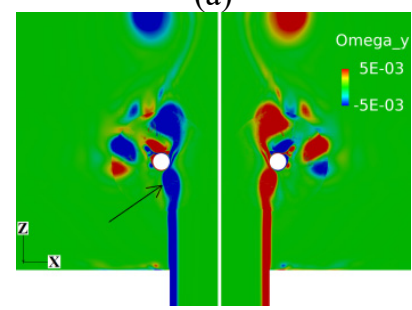

(ii) $t / t_{\mathrm{d}}=134$

(b)

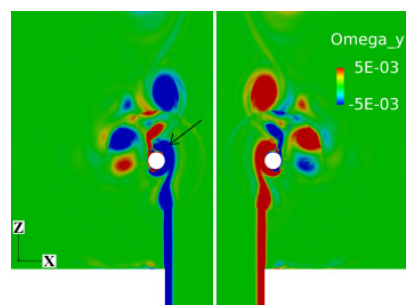

(iii) $t / t_{\mathrm{d}}=145$

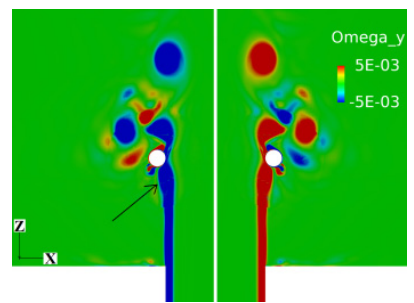

(iii) $t / t_{\mathrm{d}}=155$

Figure 11: Distribution of $\omega_{\mathrm{y}}$ when mass flows through the ring are peaks and valleys. (a) At peaks; and (b) At valleys.

Fig. 12 shows the distributions of $\mathrm{P}_{\mathrm{g}} / p_{\infty}$ when the mass flows through the rings are peaks and valleys. Although the pressure becomes high in the regions around the jet connecting the nozzle exit and the ring at the peaks, it becomes low at the valleys. Jet fluid is pushed and

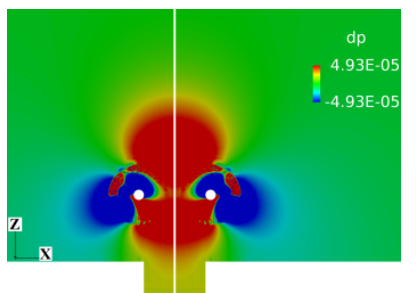

(i) $t / t_{\mathrm{d}}=72$

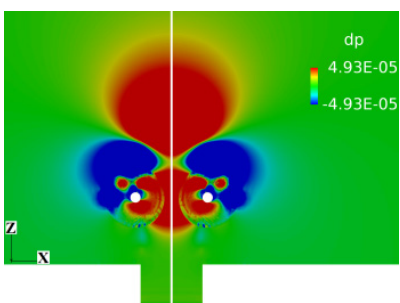

(i) $t / t_{\mathrm{d}}=95$

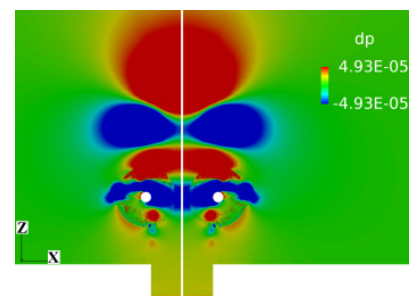

(ii) $t / t_{\mathrm{d}}=119$

(a)

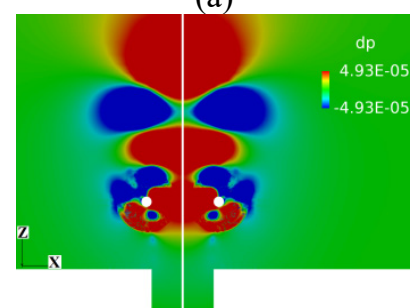

(ii) $t / t_{\mathrm{d}}=134$

(b)

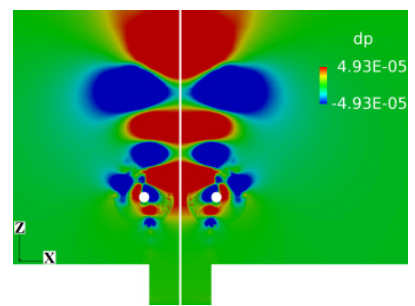

(iii) $t / t_{\mathrm{d}}=145$

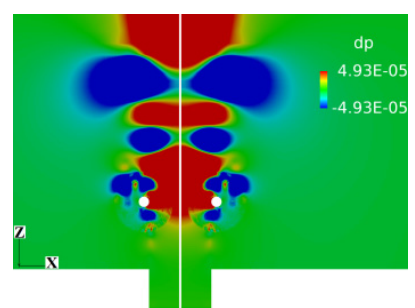

(iii) $t / t_{\mathrm{d}}=155$

Figure 12: Distribution of $\mathrm{Pg}_{\mathrm{g}} / p_{\infty}$ when mass flows through the ring are peaks and valleys. (a) At peaks; and (b) At valleys. 
mass flow through the ring becomes large when the ambient pressure is high, and jet fluid is pulled back (decelerated) and mass flow through the ring becomes small when the ambient pressure is low. Thus, this mechanism generates a self-sustained feedback oscillation.

\section{CONCLUSIONS}

Ring tone was investigated via experiments and direct sound computation. In the experiments, the frequency spectra of the ring tone were measured for various jet velocities and impingement lengths. The ring tone was also compared with the hole tone.

The peak sound intensity generally shifts to higher frequency as the jet speed increases in both tones. Multiple series of peaks are observed at a particular $L_{\mathrm{im}}$ in both tones. While the peak sound intensity generally becomes stronger as the jet speed increases, the amplification is not monotonic. When the ring tone is compared with the hole tone, the peak sound intensities are much lower and the frequency distribution is broader. The variations of the dominant ring-tone and hole-tone peak frequencies with jet speed reveal differences between the ring and hole tones at the mode jumps. Both integer stage indices and fractional indices appear.

The computation investigated the onset of the self-sustained feedback oscillation in the ring tone from the view point of the throttling mechanism. The mass flows through the ring are regulated by the variation in flow velocity and angle because of the interaction of a vortex ring around a sinuously deformed jet with the obstacle ring. This includes flow separation and the generation of secondary vortices. The mass flow becomes large when the ambient pressure in the region extending from the nozzle exit and the ring is high, and becomes small when the ambient pressure is low. Thus, the pressure fluctuation perturbs the nozzle exit where discrete vortices are generated.

\section{ACKNOWLEDGEMENTS}

Computations were partly conducted using a supercomputer system at the Japan Aerospace Exploration Agency (JAXA-JSS3). The authors thank Shin Nippon Feather Core Co., Ltd. for providing us honeycomb meshes for the wind tunnel developed in this study. The authors thank Mr. Mark Kurban from Edanz Group (https://www.jp.edanz.com/ac) for editing a draft of this manuscript.

\section{REFERENCES}

[1] Rayleigh, L., Theory of Sound, vol. 2, Dover Publication, pp. 410-412, 1945.

[2] Rockwell, D. \& Naudascher, E., Self-sustained oscillations of impinging free shear layers. Ann. Rev. Fluid Mech., 11, pp. 67-94, 1979.

[3] Blake, W.K., Mechanics of Flow-Induced Sound and Vibration, vol. 1, Academic Press, Inc., p. 151, 1986.

[4] Howe, M.S., Acoustics of Fluid-Structure Interactions, Cambridge University Press, 1998.

[5] Chanaud, R.C. \& Powell, A., Some experiments concerning the hole and ring tone. $J$. Acoust. Soc. Am., 37(5), pp. 902-911, 1965.

[6] Obata, T., Kurasawa, H. \& Haneda, Y., Self-excited oscillation in an axisymmetric jet with a coaxial ring (in Japanese). Trans. Jpn. Soc. Mech. Eng. B., 61(583), No. 94 1045, pp. 106-112, 1995.

[7] Langthjem, M.A. \& Nakano, M., A numerical simulation of the hole-tone feedback cycle based on an axisymmetric discrete vortex method and Curle's equation. J. Sound Vib., 288(1-2), pp. 133-176, 2005. 
[8] Langthjem, M.A. \& Nakano, M., A three-dimensional study of the hole-tone feedback problem. RIMS Kôkyûroku, 1697, pp. 80-94, 2010.

[9] Matsuura, K. \& Nakano, M., Direct computation of a hole-tone feedback system at very low Mach numbers. J. Fluid Sci. Tech., 6(4), pp. 548-561, 2011.

[10] Matsuura, K. \& Nakano, M., A throttling mechanism sustaining a hole tone feedback system at very low Mach numbers. J. Fluid Mech., 710, pp. 569-605, 2012.

[11] Lele, S.K., Compact finite difference schemes with spectral-like resolution. J. Comput. Phys., 103(1), pp. 16-42, 1992.

[12] Gaitonde, D.V. \& Visbal, M.R., Padé-type higher-order boundary filters for the Navier-Stokes equations. AIAA J., 38(11), pp. 2103-2112, 2000.

[13] Mukai, K. \& Matsuura, K., Experiments and computations on ring tone (in Japanese). Proceedings of the JSME, Chushikoku, 59th General Assembly Meeting, pp. 1-2, 2021.

[14] Powell, A., Some aspects of aeroacoustics: From Rayleigh until today. J. Vib. Acoust., 112, pp. 145-159, 1990.

[15] Rossiter, J.E., The effect of cavities on the buffeting of aircraft. RAE Tech. Memorandum, 754, 1962. 